\title{
Quantum chemical investigation of thermochemistry in Calvin cycle
}

\author{
DIBYENDU MONDAL, TUMPA SADHUKHAN, IQBAL A LATIF and SAMBHU N DATTA* \\ Department of Chemistry, Indian Institute of Technology - Bombay, Powai, Mumbai 400 076, India \\ e-mail: sndatta@chem.iitb.ac.in
}

MS received 6 July 2015; revised 29 August 2015; accepted 22 September 2015

\begin{abstract}
This work aims to verify the experimental thermochemistry of the reactions involved in Calvin cycle that produces glucose equivalent by using products from the light-activated reactions in chloroplast. The molecular geometry of each involved species in water has been optimized by density functional theory using SCRF-PCM methodology at M06-2X/6-311++G(3df,3pd) level. The thermal correction to Gibbs free energy of each solute has been calculated at the same level of theory. An explicit accounting of the intramolecular and intermolecular hydrogen bonding has been made for each solute molecule by using theoretically determined values from different sources. These data have been added together to obtain the standard Gibbs free energy $G^{\varnothing}$ for each molecule in solution. Finally, the free energy change $\Delta G$ of each involved reaction has been determined using the experimental concentrations under physiological conditions. The calculated $\Delta G$ values are generally in good agreement with the experimentally found free energy changes, with only a few relatively large deviations. Five regulating steps with moderately large and negative $\Delta G$ have been identified, whereas only three of them were clearly identified from experiment. We particularly show that the steps involving the formation of G3P from 3-PG and the regeneration of RuBP from Ru5P are thermodynamically strongly favored, and therefore, they take part in driving the metabolic process. We have illustrated Calvin cycle by vividly distinguishing the controlling steps from the potentially reversible reactions.
\end{abstract}

Keywords. Calvin cycle; driving force; PCM; H-bond.

\section{Introduction}

The Calvin cycle consists of the light-independent reactions of photosynthesis in green plants, which occur in stroma inside chloroplast and produce glucose equivalents. Although these reactions are known as dark reactions, they come about only when Adenosine triphosphate (ATP) and Dihydronicotinamide adenine dinucleotide phosphate (NADPH) are available, the latter being produced from Adenosine diphosphate (ADP) and Nicotinamide adenine dinucleotide phosphate $\left(\mathrm{NADP}^{+}\right)$ in the light-driven reactions taking place in and around thylakoid membrane. ATP is produced via proton-motive force in the electron transport chain whereas NADPH is the final product of the so-called $\mathrm{Z}$ scheme. The Calvin Cycle involves three basic stages ${ }^{1}$ that are illustrated in figure 1. The first step is the fixation of $\mathrm{CO}_{2}$ from inorganic forms such as the solvated $\mathrm{CO}_{2}$ molecule and the bicarbonate and carbonate ions to organic molecule such as Ribulose-1,5-bisphosphate (RuBP) in presence of enzyme RuBisco, thereby generating 3-Phosphoglyceraldehyde (3-PG). The mechanism of $\mathrm{CO}_{2}$ fixation, that is, the conversion of RuBP to $3-\mathrm{PG}$, was studied in detail by English et al. ${ }^{2}$ In the second step, ATP and

*For correspondence
NADPH reduce 3-PG into Glyceraldehyde-3-phosphate (G3P). Simultaneously, ATP converts into ADP and NADPH into NADP ${ }^{+}{ }^{3,4}$ Thisprocess leads to a chain of reactions that culminates in the production of Ribulose5-phosphate (Ru5P). The final step is on the regeneration of RuBP. Ru5P reacts with ATP in presence of enzyme Phospho-ribulokinase (PRK) and produces RuBP and ADP., ${ }^{5}$ Goldberg et al. in their series of review papers ${ }^{7-13}$ consolidated huge experimental data on thermodynamic parameters such as enthalpy change and apparent equilibrium constant of different classes of enzyme-catalyzed reactions from a large number of references.

The important steps of Calvin cycle are shown in figure 2. These are under investigation here. The original experimental work of Bassham and Krause ${ }^{1}$ was a compact study on the metabolic regulation of carbon reduction cycle which even today calls for a detailed theoretical investigation of this immensely important biochemical process. These authors investigated the stroma of Chlorella pyrenoidosa, determined the steady state concentrations of different intermediates involved in carbon reduction cycle, and found the free energy change for each step under physiological conditions. ${ }^{1}$ In spite of the elaborate experimental work done in this area, only a few quantitative treatments have been made so far. 


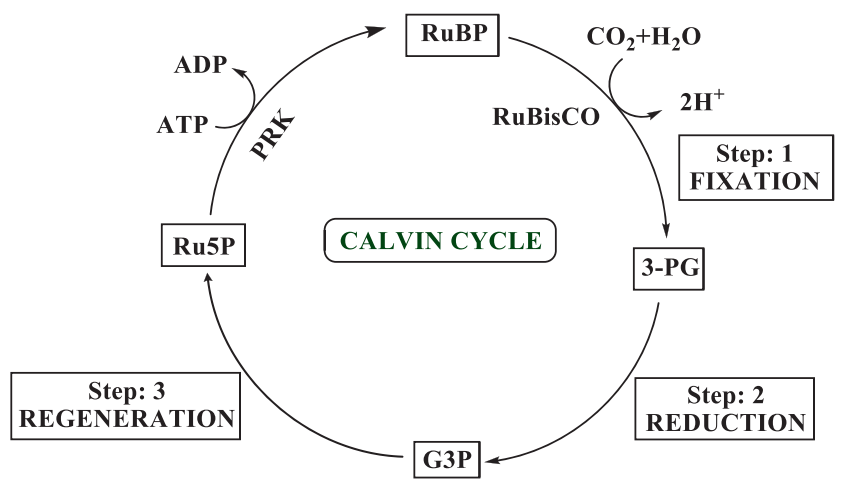

Figure 1. Schematic representation of Calvin Cycle.

Alberty has discussed a method to estimate standard transformed formation energies from the experimental data on 53 biochemical reactants. ${ }^{14}$ Leskovac et al. have used the data from the work by Alberty and Goldberg et al. estimated the free energy change for 19 enzymatic reactions corresponding to the reactions taking place in Calvin cycle and pentose phosphate pathway, under standard conditions at different $\mathrm{pH}, 25^{\circ} \mathrm{C}$ and for ionic strength $0.25 \mathrm{M} .{ }^{15}$ Both developments have been in general at the level of the "classical" physical chemistry and in particular in solution chemistry. A systematic theoretical determination of the free energy change for each involved reaction under physiological conditions is lacking, though it can generate a better understanding of the biochemical process. This has motivated the present work on quantum chemical calculation of free energy changes for the steps involved in Calvin cycle.

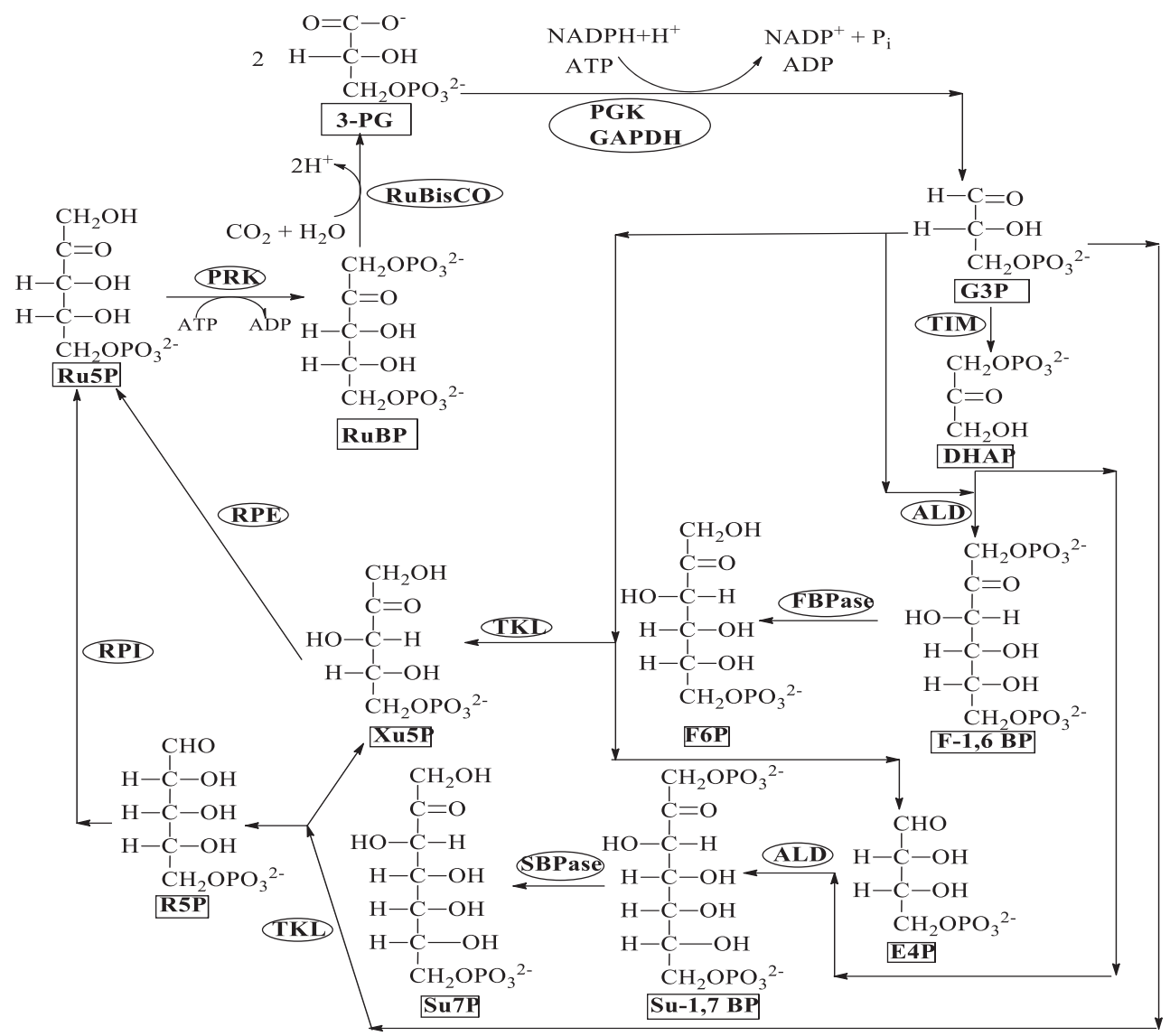

Figure 2. Steps in Calvin Cycle that are under investigation in the present work. 3-PG: 3-phosphoglycerate; ADP: Adenosine diphosphate; ATP: Adenosine triphosphate; NADPH: Dihydronicotinamide adenine dinucleotide phosphate; DHAP: Dihydroxyacetone phosphate; E4P: Erythrose-4-phosphate; ALD: Fructose-1,6-bisphosphate aldolase; FBPase: Fructose-1,6-biphosphatase; F-1,6BP: Fructose-1,6-bisphosphate; F6P: Fructose-6-phosphate; G3P: Glyceraldehyde-3-phosphate; GAPDH: Glyceraldehyde-3-phosphate dehydrogenase; NADP ${ }^{+}$: Nicotinamide adenine dinucleotide phosphate; Pi: Orthophosphate; PGK: Phosphoglycerate kinase; PRK: Phosphoribulokinase; RPI: Phosphoriboisomarase; R5P: Ribose-5-phosphate; Ru5P: Ribulose-5-Phosphate; RuBP: Ribulose-1,5-bisphosphate; RPE: Ribulose-5-Phosphate-3-Epimerase; RuBisCO: Ribulose-1,5-bisphosphate carboxylase; Su-1,7BP: Sedoheptulose-1,7-bisphosphate; Su7P: Sedoheptulose-7-phosphate; SBPase: Sedoheptulose-1,7-biphosphatase; TIM: Triosephosphateisomerase; TKL: Transketolase; Xu5P: Xylulose-5-phosphate. 
Our main objective is to verify the free energies determined by Bassham and Krauseby using quantum chemical methods. We have worked especially with density functional theory (DFT). The calculated data are important not only because they provide a justification of the processes to be spontaneously taking place in vivo as discussed by Bassham and Krause, ${ }^{1}$ but also as these and some other data can be used to successfully estimate the rate of production of glucose equivalent in $\mathrm{C}_{4}$ plants as discussed in references ${ }^{16-19}$. The present work has been designed as follows. First, we have quantum chemically calculated the Gibbs free energy $\left(G_{\text {tot }}\right)$ for the concerned species in an aqueous medium. In the second step of our work, we have added corrective terms to explicitly account for hydrogen bonding. This generates an estimate of the standard Gibbs free energy of the solvated species. In the third step, the free energy of each reaction has been determined at the "steady state" concentrations of ref. ${ }^{1}$ We have shown that to within $\pm 2 \mathrm{kcal} \mathrm{mol}^{-1}$, the calculated free energy of each reaction is in agreement with the steady state free energy change reported in ref. ${ }^{1}$ Exceptions have been noted only in a few cases.

\section{Methodology}

The initial structure for each molecule has been taken from NCBI's PubChem (http://dx.doi.org/10.1016/ S1574-1400(08)00012-1). ${ }^{20}$ The chosen PubChem CID's (also available from https://www.pubchem.ncbi. nlm.nih.gov) are as follows: 25245548 (for 3-PG), 2479 4350 (G3P), 4643300 (DHAP), 84951 (F1,6BP), 69507 (F6P), 5459820 (Xu5P), 5459862 (E4P), 164735 (Su1,7BP), 46878379 (Su7P), 77982 (R5P), 21144996 (Ru5P), 23615473 (RuBP), 5461108 (ATP), 6022 (ADP), 15983949 (NADPH) and $15938972\left(\mathrm{NADP}^{+}\right)$. The ionized forms were prepared by abstracting $\mathrm{H}^{+}$ from the acidic groups. In order to study the thermodynamics of each step at the molecular level, we have carried out density functional theory (DFT) based calculations on the involved species. As the dark reactions take place in the aqueous phase of stoma, the effect of solvent has been considered by relying on the Self-Consistent Reaction Field (SCRF) method. More explicitly, we have used the Polarizable Continuum Model (PCM) while employing the dielectric constant 78.39 for the solvent (water). This model takes care of both Born free energy of charge-dielectric interaction and Onsager free energy of dipole-dielectric interaction.

Each geometry optimization and thermal energy calculation has been done with the time-tested meta-GGA hybrid functional M06-2X. ${ }^{21,22}$ The M06-2X functional has a large amount (54\%) of the Hartree-Fock exchange and is known to produce quality data for thermochemistry. It also has an s6 scaling factor of 0.06 for Grimme's long-range dispersion correction ${ }^{21,23-26}$ which makes it ideal for usage in calculation on organic molecules with long hydrocarbon chains or backbones. A small discussion was given on the merit of M06-2X over other functional in one of our previous publications. ${ }^{27}$ The basis sets $6-311 \mathrm{G}(\mathrm{d}, \mathrm{p}), 6-311++\mathrm{G}(\mathrm{d}, \mathrm{p})$ and $6-311++\mathrm{G}(3 \mathrm{df}, 3 \mathrm{pd})$ have been successively used for the optimization of molecular geometry. Gaussian 09 (G09) suite of programs has been used for the quantum chemical calculations. ${ }^{28}$

Quantum chemically calculated free energy of solvated species can be represented by

$$
G_{\text {tot }}=E_{P C M}+G_{\text {thermal }}
$$

Where $E_{P C M}$ and $G_{\text {thermal }}$ are the electronic energy of the molecule in solution and the thermal correction to the free energy of the solvated species, respectively. The quantity $G_{\text {thermal }}$ is obtained from the calculation of rotational characteristics and vibrational frequencies in G09. It consists of statistical mechanical sums of translational, rotational and vibrational energies of the solvated molecule, the $P V$ term, and the contributions from statistical thermodynamically calculated translational, rotational and vibrational entropies, all incorporated in Gaussian code.

As PCM visualizes the system to be immersed in a dielectric continuum, the explicit correction due to hydrogen bonding $\left(G_{H-b o n d}\right)$ is missing from $G_{t o t}$. Therefore, the standard free energy of the solvated species is written as,

$$
G^{\varnothing}=G_{t o t}+G_{H-b o n d}
$$

Different types of hydrogen bonding corrections can be collected from theoretical sources. Using these data, the net hydrogen bonding corrections can be estimated from the optimized molecular structures of the species in solution, and $\Delta G^{\varnothing}$ per molecule can be determined.

The net free energy change of a reaction is given by,

$$
\Delta G=\Delta G^{\varnothing}+k T \ln Q
$$

per molecule where $Q$ is the reaction quotient. The latter quantity can be easily obtained from the observed steady state concentrations given in ref. ${ }^{1}$ The saturated concentration of carbon dioxide has been taken from Dodd's work. ${ }^{36}$ This completes the method of calculating $\Delta G$ under the experimental (physiological) conditions. 


\section{Results and Discussion}

The optimized structures for the involved species in an aqueous medium are shown in figures 3 and 4 . As we have used the ionized forms of the molecules, and because the species have been considered to be solvated, the optimized structures are generally quite different from the crystal structures of PubChem. Figures 3 and 4 show the optimized structures of the molecules in water. These are generally folded conformations, even for the smaller species like 3-PG, G3P, DHAP and E4P.

Because of their largeness, the molecular geometries of NADPH and NADP+ have been found using functional M06-2X with 6-311++G(d,p) basis set, and the same are illustrated in figure 3 . From the computed PCM energies and thermal corrections to the free energy values, we have obtained the $G_{t o t}$ of Eq. (1). The optimized structures for all other solute molecules have been determined using a much larger basis set, that is, at UM06-2X/6-311++G(3df,3pd) level. These are exhibited in figure 4. Total PCM energy calculated for each solvated molecule and the thermal correction to the corresponding free energy are given in table 1 .

Next, our focus has turned to the hydrogen bonding effects. All species involved in Calvin Cycle are stabilized due to extensive hydrogen bonding with water molecules. Different functional groups have different types of H-bonding. Each type of the H-bond energy can vary in the range $1-15 \mathrm{kcal} \mathrm{mol}^{-1}$ depending on the nature and location of the functional group. The overall contribution to free energy also depends on the extent of H-bonding due to steric restrictions. Typical $\mathrm{H}$-bond energy values are collated from references ${ }^{29-33}$ and given in table 2 where $N_{\text {bond }}$ denotes the maximum number of water molecules hydrogen bonded to the concerned type of species.
For calculating the average energy per hydrogen bond in aquated $\mathrm{Pi}$, we have adopted the following procedure. From ref. ${ }^{34}$, we have taken the $E_{I E F P C M}$ at B3LYP/ $6-311++\mathrm{G}(\mathrm{df}, \mathrm{pd})$ level for the complex $\mathrm{HPO}_{4}{ }^{2-} \cdot 9 \mathrm{H}_{2} \mathrm{O}$ (-1331.695 a.u.). We have calculated total energy for solvated orthophosphate ion $(-643.402$ a.u.) and that for water molecule in bulk water $(-76.4$ a.u.) using the same level of theory and the same basis set. The difference divided by 9 gives us the correction for each hydrogen bond, $-7.53 \mathrm{kcal} \mathrm{mol}^{-1}$, as shown in table 2 .

Reference ${ }^{29-31}$ are on gas phase calculations, while references $^{32-34}$ are on systems in aqueous medium. Because of the way the hydrogen bond energies were evaluated in references ${ }^{29-34}$, as difference between two energy values, the stability arising from the hydrogen bonding effects and the stability gained from the polarization of the dielectric medium can be considered as additive. Although the use of the values in table 2 for all other molecules constitutes an approximation, it leads to a good estimate of $\Delta G^{\varnothing}$. We write,

$$
\Delta G^{\varnothing}=\Delta G_{t o t}+\left(\sum_{i \in P} N_{i} \varepsilon_{i}-\sum_{j \in R} N_{j} \varepsilon_{j}\right)
$$

where $i$ and $j$ denote the relevant functional groups present in the products and reactants respectively, $N$ is the number of hydrogen bonds and $\varepsilon$ is the average energy per $\mathrm{H}$-bond.

A molecule with potential hydrogen bonding groups can also form intramolecular hydrogen bonds. The distance between hydrogen bonding donor and acceptor plays an important role in determining such interactions. This is where the optimized molecular structure can come into play. We have assumed that if the distance between $-(\mathrm{O}) \mathrm{H}$ and $\mathrm{O}<$ in the optimized structure of any reacting species is around $2 \AA$, then there is a possibility for intramolecular hydrogen bonding.

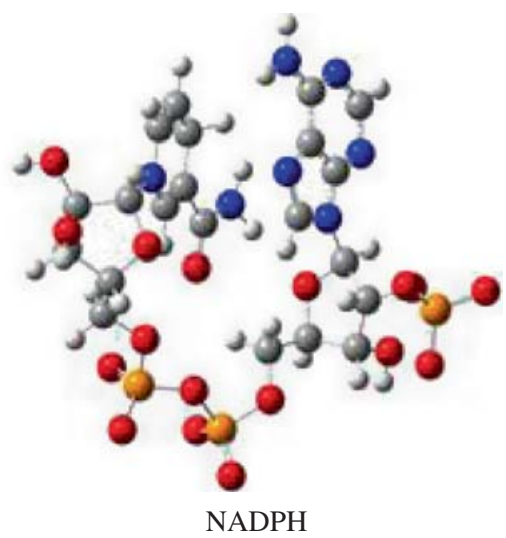

Figure 3. Molecular geometries for NADPH and NADP + in water. These have been computed at UM06-2X/6-311++G(d,p) level. 


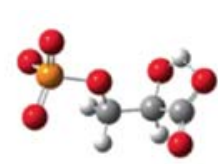

3-PG

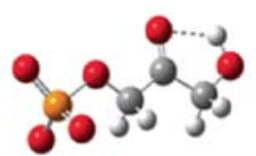

DHAP

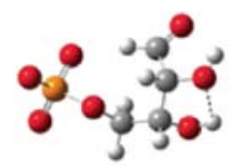

E4P
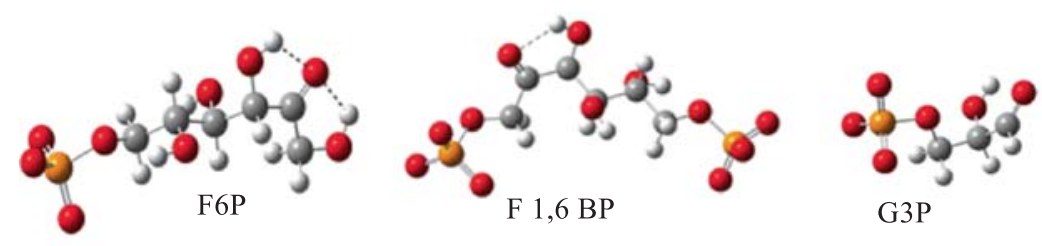

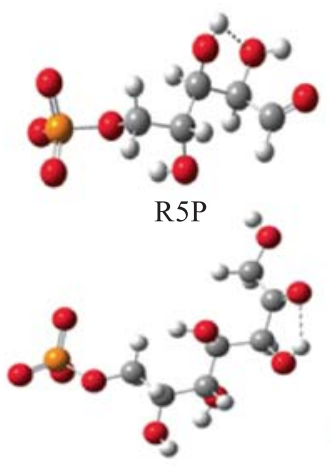

$\mathrm{Su} 7 \mathrm{P}$
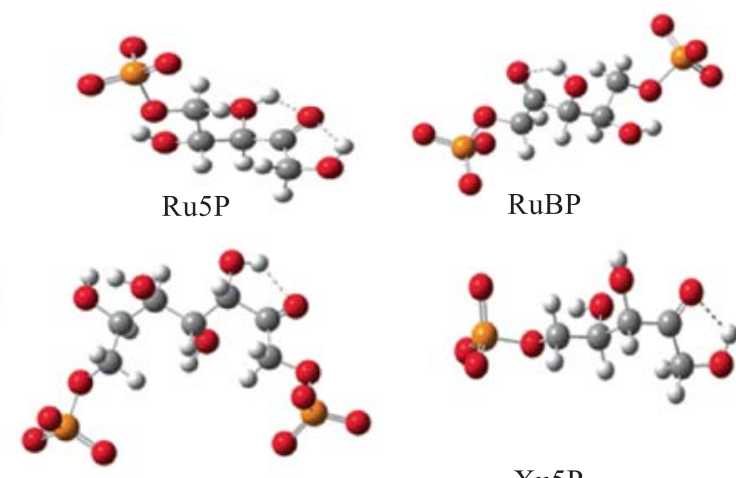

Su 1,7 BP

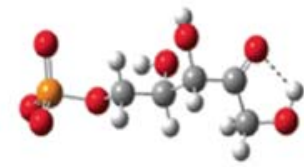

$\mathrm{Xu} 5 \mathrm{P}$

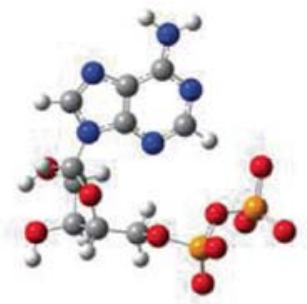

ADP

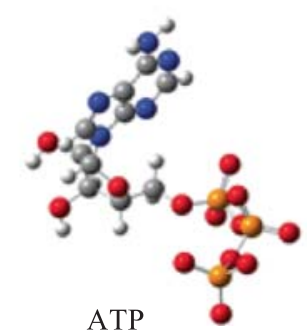

Figure 4. PCM optimized molecular geometries calculated at UM06-2X/6$311++\mathrm{G}(3 \mathrm{df}, 3 \mathrm{pd})$ level. The solute molecules are in water.

For example, the ketone group is often involved in intramolecular hydrogen bonding with an alcoholic hydroxyl group. Steric influence can hinder water molecules from approaching the carbonyl group. Hence, we have considered that in general the hydrogen bonding stability for an internal ketone group should be lower than that of acetone and approximately equal to that of aldehyde in table 2 . The aldehyde group being a terminal group is accessible to hydrogen bonding with water. Similarly, a close proximity of another alcohol group and steric congestion barring the approach of a water molecule can lead to intramolecular hydrogen bonding between vicinal alcohol groups. Phosphate and carboxylate groups would have one intramolecular hydrogen bond with an adjacent hydroxyl group when the latter bears a close proximity, and therefore, one less intermolecular hydrogen bonding has been considered in such cases. The stabilization energy for the intramolecular hydrogen bonding in these cases is taken as that for the alcoholwater complex in table 2 . The number of intermolecular hydrogen bonding with solvent molecules along with the nature and actual number of intramolecular hydrogen bonding for the optimized geometry of each particular species are given in table 3 .

Hydrogen bonding contributions to free energy of different molecules (table 3 ) has been used to calculate $\Delta G_{H \text {-bond }}$ for each reaction of Calvin cycle. Explicit hydrogen bonding contribution to the free energies of the aquated ATP and ADP have not been included in table 3 as the basic chemical structures of these two molecules differ only by a phosphate group. The optimized structure of $\operatorname{ATP}(a q$.$) in figure 4$ shows that the oxygen atom attached to the $\alpha$-positioned phosphorous is rather not accessible to the water molecule for hydrogen bonding. That is, ATP has overall four more "phosphate-type" hydrogen bonds than ADP, while Pi has nine such bindings. This observation has been taken into account whilst calculating the free energy changes for reactions (R2) and (R12) in table 4. Also, figure 3 shows the amide group to be more exposed to solvent 
Table 1. Total energy for the optimized geometry of the solvated molecule and thermal correction to free energy calculated from UM06-2X/6-311++G(3df,3pd) methodology at $298.15 \mathrm{~K}$. These calculations have been done by SCRF-PCM method and for an aqueous phase using the default dielectric constant 78.39.

\begin{tabular}{|c|c|c|c|}
\hline Molecule & $E_{P C M}$ (a.u.) & $G_{\text {thermal }}$ (a.u.) & $G_{t o t}$ (a.u.) \\
\hline 3-Phosphoglycerate (3-PG) & -985.228808 & 0.053700 & -985.175108 \\
\hline Glyceraldehyde-3-P (G3P) & -910.426430 & 0.062597 & -910.363833 \\
\hline Dihydroxy acetone-P (DHAP) & -910.432465 & 0.060693 & -910.371772 \\
\hline Fructose-1,6-P $\mathrm{P}_{2}(\mathrm{~F} 1,6 \mathrm{BP})$ & -1820.881159 & 0.152184 & -1820.728975 \\
\hline Fructose-6-P (F6P) & -1254.034497 & 0.154698 & -1253.879799 \\
\hline Xylulose-5-P (Xu5P) & -1139.500658 & 0.122956 & -1139.377700 \\
\hline Erythrose-4-P (E4P) & -1024.963667 & 0.094407 & -1024.869260 \\
\hline Sedoheptulose-1,7-P $(\mathrm{Su}-1,7 \mathrm{BP})$ & -1935.412698 & 0.184107 & -1935.228591 \\
\hline ose-7-P (Su7P) & -1368.559149 & 0.1 & -1368.374041 \\
\hline Ribose-5-P (R5P) & -1139.495651 & 0.124128 & -1139.371523 \\
\hline Ribulose-5-P (Ru5 & -1139.499537 & 0.124900 & -1139.374637 \\
\hline Ribulose-1,5-F & -1706.350005 & 0.121344 & -1706.228661 \\
\hline Adenosine triph & -2665.042613 & 0.226311 & -2664.816302 \\
\hline Adenosine diphosphate (ADP) & -2097.719701 & 0.220961 & -2097.498740 \\
\hline $\mathrm{NADPH}^{\mathrm{a}}$ & -3502.704418 & 0.465889 & -3502.238529 \\
\hline $\mathrm{NADP}^{+\mathrm{a}}$ & -3501.985968 & 0.460561 & -3501.525408 \\
\hline Carbon di & -188.586875 & -0.008805 & -188.595680 \\
\hline Phos & -643.280545 & -0.002575 & -643.283120 \\
\hline Water $\left(\mathrm{H}_{2} \mathrm{O}\right)$ & -76.429582 & 0.003845 & -76.425737 \\
\hline $\mathrm{H}_{3} \mathrm{O}^{+}$ & -76.814756 & 0.015829 & -76.798927 \\
\hline
\end{tabular}

${ }^{a}$ At UM06-2X/6-311++G(d,p) level using PCM and 298.15 K.

in NADPH than in $\mathrm{NADP}^{+}$. Thus, the maximum hydrogen bonding stability of NADPH relative to $\mathrm{NADP}^{+}$ can be estimated to be about $20 \mathrm{kcal} \mathrm{mol}^{-1}$ accounting for two carbonyl-water bonds, four $(\mathrm{N}) \mathrm{H}$-water bonds, and two $(\mathrm{H}) \mathrm{N}$-water bonds. In reality the amide group in $\mathrm{NADP}^{+}$is partially exposed and that in NADPH is

Table 2. Average hydrogen bonding energy $(\epsilon)$ and the number of hydrogen bonds $\left(N_{b o n d}\right)$ for species with different functional groups.

\begin{tabular}{lccc}
\hline Hydrogen bonded System & $\epsilon\left(\mathrm{kcal} \mathrm{mol}^{-1}\right)$ & $N_{\text {bond }}$ & Ref. \\
\hline $\mathrm{H}_{2} \mathrm{O} \cdots \mathrm{H}-\mathrm{OH}^{\mathrm{a}}$ & $\epsilon_{1}=-2.39$ & 4 & 29 \\
$\mathrm{H}_{2} \mathrm{CO} \cdots \mathrm{H}-\mathrm{OH}^{\mathrm{a}}$ & $\epsilon_{2}=-2.15$ & 2 & 29 \\
$\left(\mathrm{CH}_{3}\right)_{2} \mathrm{CO} \cdots \mathrm{H}-\mathrm{OH}^{\mathrm{a}}$ & $\epsilon_{3}=-3.61$ & 2 & 29 \\
$\mathrm{C}_{2} \mathrm{H}_{5} \mathrm{O}(\mathrm{H}) \cdots \mathrm{H}-\mathrm{OH}^{\mathrm{b}}$ & $\epsilon_{4}=-3.29$ & 3 & 30 \\
$\mathrm{H}_{2} \mathrm{O} \cdots \mathrm{HOC}_{2} \mathrm{H}_{5}^{\mathrm{b}}$ & & & \\
$\mathrm{H}-\mathrm{O}-\mathrm{H} \cdots \mathrm{CO}_{2}{ }^{\mathrm{c}}$ & $\epsilon_{5}=-2.47$ & 1 & 31 \\
$\mathrm{C}(\mathrm{O}) \mathrm{O}^{-} \cdots \mathrm{H}_{-} \mathrm{O}-\mathrm{H}$ & $\epsilon_{6}=-13.6$ & 3 & 32 \\
$\mathrm{H}_{2} \mathrm{O} \cdots \mathrm{H}_{3} \mathrm{O}^{+\mathrm{d}}$ & $\epsilon_{8}=-3.58$ & 3 & 33 \\
$\mathrm{HPO}_{4}^{2-} \cdots \mathrm{H}_{-}-\mathrm{O}-\mathrm{H}^{\mathrm{e}}$ & $\epsilon_{7}=-7.53$ & 9 & 34 \\
$\mathrm{H}_{2} \mathrm{O} \cdots \mathrm{HPO}_{4}{ }^{2-\mathrm{e}}$ & & & \\
\hline
\end{tabular}

${ }^{\text {a }}$ The quantity $\epsilon$ was estimated as $\Delta \mathrm{E}(\mathrm{T})-\mathrm{BSSE}(\mathrm{T})-\Delta \mathrm{ZPVE}(\mathrm{D})$ using HF, B3LYP and MP2 levels with the basis aug-cc-pvDZ(D) and aug-ccpvTZ(T) $\left(\right.$ ref. $\left.^{29}\right)$.

${ }^{\mathrm{b}}$ For alcohol-water system we have considered the average of the two types of $\mathrm{H}$-bonding energies like $\mathrm{X}-\mathrm{H} \cdots \mathrm{OH}_{2}$ and $\mathrm{HO}-\mathrm{H} \cdots \mathrm{XH}$ where DA stands for proton donor and acceptor, respectively. We have considered two WE and one EW in our calculation on the water-ethanol system $\left(\right.$ ref. $\left.^{30}\right)$.

${ }^{\mathrm{C}}$ Only the most stable T-shaped configuration is considered (ref. ${ }^{31}$ ). ${ }^{\mathrm{d}}$ Hydrogen atoms of $\mathrm{H}_{3} \mathrm{O}^{+}$are only involved in hydrogen bonding (ref. ${ }^{33}$ ).

${ }^{\mathrm{e}}$ As discussed in text, using the data from ref. ${ }^{34}$ 
Table 3. Estimation of the contribution of hydrogen bonding to standard free energy of the solvated species.

\begin{tabular}{|c|c|c|c|c|}
\hline \multirow[b]{3}{*}{ Species } & \multicolumn{3}{|c|}{ Hydrogen bonding } & \multirow{3}{*}{$\begin{array}{l}\text { Contribution } \\
\text { to } G^{\varnothing} \\
\left(\mathrm{kcal} \mathrm{mol}^{-1}\right)\end{array}$} \\
\hline & \multirow{2}{*}{$\begin{array}{c}\text { Total } \\
\text { Number }\end{array}$} & \multicolumn{2}{|c|}{ Intramolecular } & \\
\hline & & Number & Nature $^{\mathrm{a}, \mathrm{b}, \mathrm{c}, \mathrm{d}}$ & \\
\hline 3-Phosphoglycerate(3-PG) & 14 & 1 & $(\mathrm{CO}) \mathrm{O} \cdot \cdot \mathrm{HO}_{-}$ & -97.31 \\
\hline Glyceraldehyde-3-P (G3P) & 13 & 0 & - & -74.41 \\
\hline Dihydroxy acetone-P (DHAP) & 13 & 1 & $(\mathrm{C}) \mathrm{O} \cdots \mathrm{HO}-$ & -72.26 \\
\hline Fructose-1,6- $\mathrm{P}_{2}(\mathrm{~F} 1,6 \mathrm{BP})$ & 27 & 1 & (C)O $\cdots \mathrm{HO}_{-}$ & -152.24 \\
\hline Fructose-6-P (F6P) & 22 & 2 & (C)O $\cdots \mathrm{HO}_{-}$ & -99.72 \\
\hline Xylulose-5-P (Xu5P) & 19 & 3 & $\begin{array}{c}2(\mathrm{C}) \mathrm{O} \cdots \mathrm{HO}- \\
(\mathrm{P}) \mathrm{O} \cdots \mathrm{HO}-\end{array}$ & -86.56 \\
\hline Erythrose-4-P (E4P) & 16 & 1 & $(\mathrm{H}) \mathrm{O} \cdots \mathrm{HO}-$ & -80.99 \\
\hline Sedoheptulose-1,7- $\mathrm{P}_{2}(\mathrm{Su}-1,7 \mathrm{BP})$ & 30 & 1 & (C)O $\cdots \mathrm{HO}_{-}$ & -162.11 \\
\hline Sedoheptulose-7-P (Su7P) & 23 & 3 & $\begin{array}{c}2(\mathrm{C}) \mathrm{O} \cdots \mathrm{HO}- \\
(\mathrm{H}) \mathrm{O} \cdots \mathrm{HO}-\end{array}$ & -106.30 \\
\hline Ribose-5-P (R5P) & 19 & 1 & $(\mathrm{H}) \mathrm{O} \cdots \mathrm{HO}-$ & -90.86 \\
\hline Ribulose-5-P (Ru5P) & 19 & 2 & $(\mathrm{C}) \mathrm{O} \cdots \mathrm{HO}-$ & -89.85 \\
\hline Ribulose-1,5- $\mathrm{P}_{2}$ (RuBP) & 24 & 1 & $(\mathrm{C}) \mathrm{O} \cdots \mathrm{HO}-$ & -142.37 \\
\hline
\end{tabular}

${ }^{\mathrm{a}}(\mathrm{C}) \mathrm{O} \cdots \mathrm{HO}-$ denotes intramolecular hydrogen bonding between carbonyl oxygen and adjacent hydroxyl group.

${ }^{b}(\mathrm{H}) \mathrm{O} \cdots \mathrm{HO}-$ denotes intramolecular hydrogen bonding between oxygen of an alcohol group with adjacent hydroxyl group.

${ }^{\mathrm{c}}(\mathrm{CO}) \mathrm{O} \cdots \mathrm{HO}-$ denotes intramolecular hydrogen bonding between carboxylate oxygen and adjacent hydroxyl group.

${ }^{\mathrm{d}}(\mathrm{P}) \mathrm{O} \cdots \mathrm{HO}-$ denotes intramolecular hydrogen bonding between oxygen atom attached to the phosphorous with the adjacent hydroxyl group.

partially hidden. At most only half of this data $(10 \mathrm{kcal}$ $\mathrm{mol}^{-1}$ ) can be used for the estimation of free energy of step (R2). The calculated standard free energy changes for various reactions are given in this table.

From table 4 it is evident that our calculated $\Delta G^{\varnothing}$ is generally in agreement with $\Delta G^{\prime}$ obtained from ref. $^{1}$, especially considering that such small values are extremely difficult to simulate from hard core abinitio quantum chemical calculations involving certain approximations in the guise of a chosen functional and the basis set truncation error. Somewhat large deviations are noticed only for reactions (R2), (R5) and (R12). For a negative $\Delta G^{\prime}$, we have always obtained a negative $\Delta G^{\varnothing}$. Corresponding to a small positive $\Delta G^{\prime}$,

Table 4. Calculation of Standard free energies of reaction $\left(\Delta G^{\varnothing}\right.$ in $\left.\mathrm{kcal} \mathrm{mol}^{-1}\right)$ in aqueous phase (dielectric constant 78.39). We have used SCRF-PCM method, UM06-2X hybrid functional and 6-311++G (3df, 3pd) basis set.

\begin{tabular}{|c|c|c|c|c|c|}
\hline & Reaction & $\Delta G_{t o t}$ & $\Delta G_{H-b o n d}$ & Calc. $\Delta G^{\varnothing}$ & Obs. $\Delta G^{\prime a}$ \\
\hline (R1) & $\mathrm{CO}_{2}+\mathrm{RuBP}+3 \mathrm{H}_{2} \mathrm{O} \rightarrow 23-\mathrm{PG}+2 \mathrm{H}_{3} \mathrm{O}^{+}$ & $32.68^{b}$ & -40.22 & -7.54 & -8.3 \\
\hline (R2) & $3-\mathrm{PG}+\mathrm{ATP}+\mathrm{NADPH}+\mathrm{H}_{3} \mathrm{O}^{+} \rightarrow \mathrm{ADP}+\mathrm{G} 3 \mathrm{P}+\mathrm{NADP}^{+}+\mathrm{H}_{2} \mathrm{O}+\mathrm{Pi}$ & $-10.83^{b}$ & $-4.75^{c}$ & $-15.58^{c}$ & -4.3 \\
\hline (R3) & $\mathrm{G} 3 \mathrm{P} \rightarrow \mathrm{DHAP}$ & -4.98 & 2.15 & -2.83 & -1.8 \\
\hline (R4) & $\mathrm{G} 3 \mathrm{P}+\mathrm{DHAP} \rightarrow \mathrm{F}-1,6 \mathrm{BP}$ & 4.16 & -5.57 & -1.41 & -5.2 \\
\hline (R5) & $\mathrm{F}-1,6 \mathrm{BP}+\mathrm{H}_{2} \mathrm{O} \rightarrow \mathrm{F} 6 \mathrm{P}+\mathrm{Pi}$ & -5.15 & -5.69 & -10.84 & -3.4 \\
\hline (R6) & $\mathrm{G} 3 \mathrm{P}+\mathrm{F} 6 \mathrm{P} \rightarrow \mathrm{Xu} 5 \mathrm{P}+\mathrm{E} 4 \mathrm{P}$ & -2.09 & 6.58 & 4.49 & +1.5 \\
\hline (R7) & $\mathrm{DHAP}+\mathrm{E} 4 \mathrm{P} \rightarrow \mathrm{Su}-1,7 \mathrm{BP}$ & 7.81 & -8.86 & -1.05 & -5.6 \\
\hline (R8) & $\mathrm{Su}-1,7 \mathrm{BP}+\mathrm{H}_{2} \mathrm{O} \rightarrow \mathrm{Su} 7 \mathrm{P}+\mathrm{Pi}$ & -1.78 & -2.40 & -4.18 & -3.4 \\
\hline (R9) & $\mathrm{G} 3 \mathrm{P}+\mathrm{Su} 7 \mathrm{P} \rightarrow \mathrm{R} 5 \mathrm{P}+\mathrm{Xu} 5 \mathrm{P}$ & -7.12 & 3.29 & -3.83 & +0.1 \\
\hline (R10) & $\mathrm{Xu} 5 \mathrm{P} \rightarrow \mathrm{Ru} 5 \mathrm{P}$ & 1.92 & -3.29 & -1.37 & +0.2 \\
\hline (R11) & $\mathrm{R} 5 \mathrm{P} \rightarrow \mathrm{Ru} 5 \mathrm{P}$ & -1.95 & 1.01 & -0.94 & +0.5 \\
\hline$(\mathrm{R} 12)$ & $\mathrm{Ru} 5 \mathrm{P}+\mathrm{ATP}+\mathrm{H}_{2} \mathrm{O} \rightarrow \mathrm{RuBP}+\mathrm{ADP}+\mathrm{H}_{3} \mathrm{O}^{+}$ & $24.88^{b}$ & -22.40 & +2.48 & -5.2 \\
\hline
\end{tabular}

${ }^{a}$ Standard physiological free energy change of the reaction from ref. ${ }^{1}$

${ }^{b}$ Using the experimental heat of hydration of proton $-266.0 \mathrm{kcal} \mathrm{mol}^{-1}$ from ref. ${ }^{35}$

${ }^{c}$ Assuming hydrogen bonding energy difference between NADP ${ }^{+}$and NADPH is $10.0 \mathrm{kcal} / \mathrm{mol}$. 
Table 5. Experimental concentrations of different species from ref. ${ }^{1}$

\begin{tabular}{lc}
\hline Solute & Concentration $(\mathrm{mM})$ \\
\hline 3-Phosphoglycerate (3-PG) & 1.400 \\
Glyceraldehyde-3-P (G3P) & 0.032 \\
Dihydroxy acetone-P (DHAP) & 0.640 \\
Fructose-1,6-P (F1,6BP) & 0.097 \\
Fructose-6-P (F6P) & 0.530 \\
Xylulose-5-P (Xu5P) & 0.021 \\
Erythrose-4-P (E4P) & 0.020 \\
Sedoheptulose-1,7-P2 (Su-1,7BP) & 0.114 \\
Sedoheptulose-7-P (Su7P) & 0.248 \\
Ribose-5-P (R5P) & 0.034 \\
Ribulose-5-P (Ru5P) & 0.012 \\
Ribulose-1,5-P $(\mathrm{RuBP})$ & 2.040 \\
Adenosine diphosphate (ADP)/ & $1: 3$ \\
Adenosine triphosphate (ATP) & $33.6^{a}$ \\
Carbon di-oxide (CO $\left.{ }_{2}\right)$ & 1.000 \\
[NADPH]/[NADP $^{+}$] & 1.000 \\
Phosphate (Pi) & $2.00 \times 10^{-5}$ \\
$\mathrm{H}_{3} \mathrm{O}^{+}$ & \\
\hline
\end{tabular}

${ }^{a}$ From ref. ${ }^{36}$

we have obtained either a small positive or a small negative $\Delta G^{\varnothing}$. The credit for this success goes to the DFT methodology and the functional M06-2X that is reputed for reproducing benchmark thermochemistry.

To obtain the free energy changes under experimental steady state conditions, we have used steady state concentrations of different intermediates from ref. ${ }^{1}$ As the concentration of $\mathrm{CO}_{2}$ given in this reference is too low and likely to be a printing mistake, we have adopted the saturated concentration from ref. ${ }^{36}$ The chosen concentrations are collated in table 5 for a ready reference.

Since the steady state free energy change of a reaction is more important than the standard free energy change for considering the reversibility, we have used equation (3) to calculate the $\Delta G$ for each reaction and compared it with the experimental value in table 6 . In most of the cases, the calculated $\Delta G$ agrees with the experimental data within $\pm 2 \mathrm{kcal} / \mathrm{mol}$. Large deviations have been found only for reactions (R1), (R2) and (R5). The most prominent deviation is shown by $(\mathrm{R} 1)$. This is understandable as the concentration of $\mathrm{CO}_{2}$ used in ref. ${ }^{1}$ was too low $\left(0.4 \times 10^{-3} \mathrm{~atm}\right.$. or $\left.0.01635 \mathrm{mM}\right)$. Use of the known solubility $(33.6 \mathrm{mM})$ would lead to an improved experimental $\Delta G^{s}$ of $-14.3 \mathrm{kcal} \mathrm{mol}^{-1}$ that has a smaller difference from our calculated value $-30.6 \mathrm{kcal} \mathrm{mol}^{-1}$. Still, the much lower values calculated for $\Delta G$ of reactions (R1), (R2) and (R5) lead us to conclude that these three reactions indeed would have large negative free energy changes.

Table 6 also clarifies that the reactions involving enzymes such as RuBisCO, PGK and GADPH, FBPase, SBPase and PRK, that is, reactions (R1), (R2), (R5), (R8) and (R12), are the driving force under experimental conditions. This is so because the reactions with high negative free energy changes are mainly unidirectional and they propel metabolic regulation, whereas reactions having small negative or positive values for the free energy change often take place in a reversible manner. In particular, reactions (R1), (R2) and (R5) appear to very strongly regulate the Calvin cycle. This contrasts with the observation of Bassham and Krause ${ }^{1}$ that

Table 6. Free energies of reaction in $\mathrm{kcal} \mathrm{mol}^{-1}$ at steady state concentrations of different reacting species. Steps with $\Delta G<-6 \mathrm{kcal} / \mathrm{mol}$ are regulating and they are indicated by $\boldsymbol{R}$.

\begin{tabular}{|c|c|c|c|c|}
\hline & Reaction & $R T \ln Q$ & $\Delta G(\text { calc. })^{a}$ & $\Delta G^{s}\left(\right.$ ref. $\left.^{1}\right)$ \\
\hline (R1) & $\mathrm{CO}_{2}+\mathrm{RuBP}+3 \mathrm{H}_{2} \mathrm{O} \rightarrow 23-\mathrm{PG}+2 \mathrm{H}_{3} \mathrm{O}^{+}$ & -23.11 & $-30.65 R$ & $\begin{array}{l}-9.8 \pm 1 \boldsymbol{R} \\
-14.3 \pm 1^{b}\end{array}$ \\
\hline (R2) & $3-\mathrm{PG}+\mathrm{ATP}+\mathrm{NADPH}+\mathrm{H}_{3} \mathrm{O}^{+} \rightarrow \mathrm{ADP}+\mathrm{G} 3 \mathrm{P}+\mathrm{NADP}^{+}+\mathrm{H}_{2} \mathrm{O}+\mathrm{Pi}$ & 3.52 & $-12.06 \boldsymbol{R}$ & $-1.6 \pm 1$ \\
\hline (R3) & $\mathrm{G} 3 \mathrm{P} \rightarrow \mathrm{DHAP}$ & 1.78 & -1.05 & $-0.2 \pm 1$ \\
\hline (R4) & $\mathrm{G} 3 \mathrm{P}+\mathrm{DHAP} \rightarrow \mathrm{F}-1,6 \mathrm{BP}$ & 5.01 & 3.60 & $-0.4 \pm 1$ \\
\hline (R5) & $\mathrm{F}-1,6 \mathrm{BP}+\mathrm{H}_{2} \mathrm{O} \rightarrow \mathrm{F} 6 \mathrm{P}+\mathrm{Pi}$ & -3.09 & $-13.93 \boldsymbol{R}$ & $-6.5 \pm 1 \boldsymbol{R}$ \\
\hline (R6) & $\mathrm{G} 3 \mathrm{P}+\mathrm{F} 6 \mathrm{P} \rightarrow \mathrm{Xu} 5 \mathrm{P}+\mathrm{E} 4 \mathrm{P}$ & -2.19 & 2.30 & $-0.9 \pm 1$ \\
\hline (R7) & $\mathrm{DHAP}+\mathrm{E} 4 \mathrm{P} \rightarrow \mathrm{Su}-1,7 \mathrm{BP}$ & 5.39 & 4.34 & $-0.2 \pm 1$ \\
\hline (R8) & $\mathrm{Su}-1,7 \mathrm{BP}+\mathrm{H}_{2} \mathrm{O} \rightarrow \mathrm{Su} 7 \mathrm{P}+\mathrm{Pi}$ & -3.63 & $-7.81 \boldsymbol{R}$ & $-7.1 \pm 1 \boldsymbol{R}$ \\
\hline (R9) & $\mathrm{G} 3 \mathrm{P}+\mathrm{Su} 7 \mathrm{P} \rightarrow \mathrm{R} 5 \mathrm{P}+\mathrm{Xu} 5 \mathrm{P}$ & -1.43 & -5.26 & $-1.4 \pm 1$ \\
\hline (R10) & $\mathrm{Xu} 5 \mathrm{P} \rightarrow \mathrm{Ru} 5 \mathrm{P}$ & -0.33 & -1.70 & $-0.1 \pm 1$ \\
\hline (R11) & $\mathrm{R} 5 \mathrm{P} \rightarrow \mathrm{Ru} 5 \mathrm{P}$ & -0.62 & -1.56 & $-0.1 \pm 1$ \\
\hline (R12) & $\mathrm{Ru} 5 \mathrm{P}+\mathrm{ATP}+\mathrm{H}_{2} \mathrm{O} \rightarrow \mathrm{RuBP}+\mathrm{ADP}+\mathrm{H}_{3} \mathrm{O}^{+}$ & -8.11 & $-5.63 R$ & $-3.8 \pm 1 \boldsymbol{R}^{\prime c}$ \\
\hline
\end{tabular}

${ }^{a} \Delta G=\Delta G^{\varnothing}+R T \ln Q$.

${ }^{b}$ After correcting for $\mathrm{CO}_{2}$ concentration.

${ }^{c}$ Possibility of being partially in control (indicated by prime) was tentatively suggested in ref. ${ }^{1}$ 
reaction (R2) may not be a regulating step while reaction (R12) may be partially in control.

The reason for the large (negative) free energy associated with reaction (R1) is the strong hydrogen bonding found for the rather small molecule ion $3-\mathrm{PG}^{3-}$ with charge -3 . Similarly, the large (negative) $\Delta G$ calculated for reaction (R2) arises from the strong intramolecular hydrogen bonding in solvated $\mathrm{Pi}\left[\mathrm{HPO}_{4}{ }^{2-} .9 \mathrm{H}_{2} \mathrm{O}\right.$ (aq.)]. The same phenomenon has been observed for reactions (R5) and (R8). For reaction (R8), the slightly large PCM stability of Su-1,7BP in its bent conformation (figure 4) partially counters this effect and produces an overall $\Delta G$ of $-7.8 \mathrm{kcal} / \mathrm{mol}$ (observed: $-7.1 \pm$ $1 \mathrm{kcal} / \mathrm{mol}$ ).

Considering the estimated free energy in table 6, we present the Calvin cycle in a slightly modified form in figure 5 where the strongly irreversible steps are shown as bold red arrows, while the reversible conversions are indicated by dashed blue lines.

There are several obvious pitfalls in such complicated calculations. One is that we have optimized all structures in water medium having dielectric constant 78.39, and some amount of error can occur as the exact dielectric constant of stroma fluid is unknown. The volume of the fluid is an important factor too, as the aqueous part is contained within membrane in a fibrous and protein environment so that the assumption of a uniform dielectric continuum becomes questionable. As most of the reactants and products are charged organic molecules, newer complications arise. Whereas an electrolyte can increase the dielectric constant of the solution, a covalent organic moiety will have the opposite effect. The present work has been conducted assuming that these two opposite effects nullify each other,

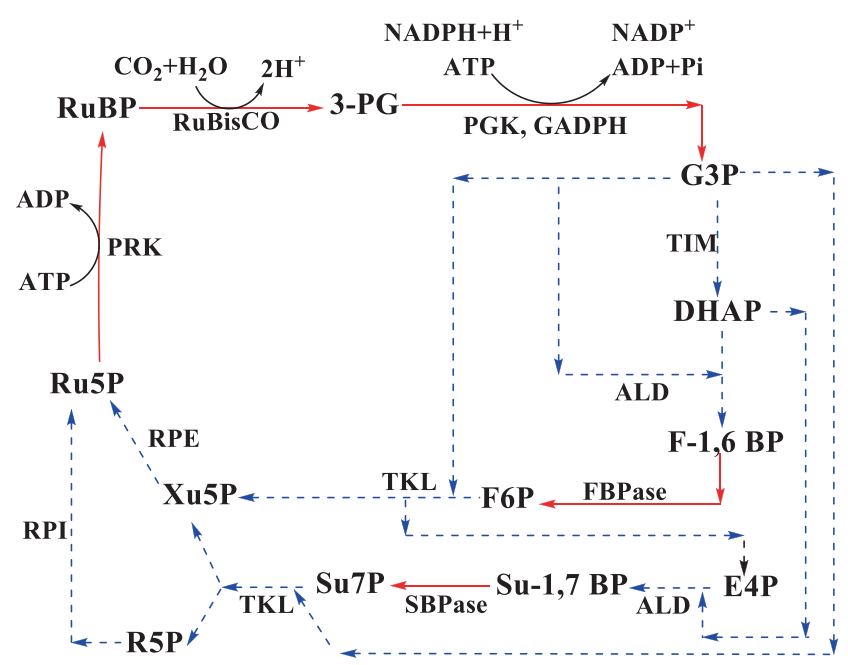

Figure 5. The Calvin cycle redrawn: the controlling steps are shown in bold and red, while potential reversible steps are indicated by dashed blue lines. especially because the concentrations involved are low enough to cause only minor changes in the dielectric constant.

Another drawback is to rely on a functional and a finite basis set, which always gives rise to inherent errors in a calculation. Nevertheless, in most cases the error in the product energies cancels the error in the energy of reactants so that the energy change is more or less correctly reproduced when a good theoretical methodology is in use along with a sufficiently large basis set.

The third problem arises from an estimation of the hydrogen bond corrections. This is certainly not an easy job, and we have adopted theoretical values from a number of sources. But there is a fallacy: the average alcoholic hydrogen bond energy differs, albeit slowly, from methanol to ethanol to glycol to, so to say, fructose and glucose..$^{30,37}$ Also, these values were obtained by using different methods employing different basis sets.

In spite of these limitations, reactions (R1), (R2) and (R5) are seen to govern the Calvin cycle while reactions (R8) and (R12) provide good support. Figure 5 gives a better energetic representation of this metabolic process.

\section{Conclusions}

The free energy change accompanying each reaction has been estimated in four steps. First, we have optimized the geometry of each solute molecule in water by using the SCRF-PCM methodology. Quantum chemical calculations have been done at the DFT level using the new versatile functional M06-2X and the really extensive $6-311++\mathrm{G}(3 \mathrm{df}, 3 \mathrm{pd})$ basis set. A vibrational frequency analysis and estimation of the thermal correction to free energy have been achieved by using FREQ code word in G09. As the PCM method fundamentally gives an estimate of Born free energy of charge-dielectric interaction and Onsager free energy of dipole-dielectric interaction, an explicit accounting for the hydrogen bonding is evidently required. The hydrogen bonding corrections for specific types of functional groups and molecules have been gathered from different sources, and in one case (for Pi), the hydrogen bonding correction has been estimated as the difference of SCRF-PCM energy between the solvated complex $\mathrm{HPO}_{4}{ }^{2-} \cdot 9 \mathrm{H}_{2} \mathrm{O}$ and the sum of energies for the solvated ion $\mathrm{HPO}_{4}^{2-}$ plus 9 solvated water molecules in bulk water. Then we have calculated the $\Delta G^{\varnothing}$ for each reaction. Addition of the term $k T \ln Q$ has reproduced the free energy changes under experimental (physiological) conditions at $25^{\circ} \mathrm{C}$. These estimated $\Delta G$ values are 
given in table 6 , and are, in principle, in agreement with the values experimentally determined by Bassham and Krause. ${ }^{1}$ A better thermochemical representation of the cycle has been obtained as figure 5. The general limitations of these calculations have been discussed. It is transparent that reactions (R1), (R2) and (R5) are the main drivers of the Calvin cycle, while being assisted by reactions (R8) and (R12).

The present calculations make sense as without the step (R2) in the driver's seat, G3P will always be in short supply, which will severely curtail the steady state concentration of G3P that is involved in a number of subsequent steps, and therefore, severely restrict the rate of production of glucose equivalent. Furthermore, without the highly spontaneous nature of (R12), the metabolic process cannot efficiently proceed as some of the earlier steps are quite reversible.

\section{Supplementary Information}

Content includes the Gaussian 09 logfiles for SCRFPCM geometry optimization and frequency calculation on all solvated systems, and ref. ${ }^{28}$ in full. Supplementary Information is available at www.ias.ac.in/ chemsci.

\section{Acknowledgments}

SND is grateful to Council of Scientific and Industrial Research for financial support (Grant No. 01/2481/11/EMR-II). We acknowledge I.I.T. Bombay computer center for their generous support and facilities.

\section{References}

1. Bassham J A and Krause G H 1969 Biochim. Biophys. Acta 189207

2. El-Hendawy M M, Garate J A, English N J, O'Reilly S and Mooney D A 2012 J. Chem. Phys. 137145103 (1-10)

3. Fridlyand L E 1992 Biochim. Biophys. Acta 1102115

4. Fridlyand L E, Backhausen J E, Holtgrefe S, Kitzmann C and Scheibe R 1997 Plant Cell Physiol. 381177

5. Runquist J A, Ríos S E, Vinarov D A and Miziorko H M 2001 Biochemistry 4014530

6. Runquist J A and Miziorko H M 2006 Prot. Sci. 15837

7. Goldberg R N, Tewari Y B, Bell D, Fazio K and Anderson E 1993 J. Phys. Chem. Ref. Data. 22530

8. Goldberg R N and Tewari Y B 1994 J. Phys. Chem. Ref. Data. 23547
9. Goldberg R N and Tewari Y B 1994 J. Phys. Chem. Ref. Data. 231035

10. Goldberg R N and Tewari Y B 1995 J. Phys. Chem. Ref. Data. 241669

11. Goldberg R N and Tewari Y B 1995 J. Phys. Chem. Ref. Data. 241765

12. Goldberg R N 1999 J. Phys. Chem. Ref. Data. 28931

13. Goldberg R N, Tewari Y B and Bhat T N 2007 J. Phys. Chem. Ref. Data. 361347

14. Alberty R A 1998 Arch. Biochem. Biophys. 353116

15. Leskovac V, Trivić S, Periči D, Popović $M$ and Kandrač J 2008 Indian J. Biochem. Biophys. 45157

16. Mehta N, Panda A, Sinha Roy A and Datta S N 2007 J. Phys. Chem. B 111919

17. Panda A and Datta S N 2007 J. Chem. Sci. 119449

18. Panda A, Bhattacharyya S and Datta S N 2009 J. Chem. Sci. 121535

19. (a) Mehta N, Panda A, Sengupta S and Datta S N 2006 J. Phys. Chem. B 110 10951; (b) Erratum: 2006 J. Phys. Chem. B 11014524

20. Bolton E, Wang Y, Theissen P A and Bryant S H 2008 Annu. Rep. Comput. Chem. 4217

21. Zhao Y and Truhlar D G 2008 Theor. Chem. Acc. 120 215

22. Zhao Y and Truhlar D G 2008 Acc. Chem. Res. 41157

23. Liu Y, Zhao J, Li F and Chen Z 2013 J. Comput. Chem. 34121

24. Walker M, Harvey A J A and Sen A 2013 J. Phys. Chem. A 11712590

25. Mardirossian N, Lambrecht D S, McCaslin L, Xantheas S S and Head-Gordon M 2013 J. Chem. Theor. Comput. 91368

26. Jones G J, Robertazzi A and Platts J A 2013 J. Phys. Chem. B 1173315

27. Sadhukhan T, Latif I A and Datta S N 2014 J. Phys. Chem. B 1188782

28. Frisch M J, et al. 2009 Gaussian 09, Revision A.02 (Wallingford CT: Gaussian, Inc.)

29. Fileti E E, Chaudhuri P and Canuto S 2004 Chem. Phys. Lett. 400494

30. Vila A and Mosquera R A 2003 Chem. Phys. 29173

31. Sadlej J and Mazurek P 1995 J. Mol. Struc. Theochem. 337129

32. Jorgensen W L and Gao J 1986 J. Phys. Chem. 902174

33. Markovitch O and Agmon N 2007 J. Phys. Chem. A 111 2253

34. Paul W A 2007 In Molecular Modeling Study of Sulfate and Phosphate Adsorption at the Mineral-Water Interface PhD dissertation (Newark, Delaware, USA: University of Delaware)

35. Bockris J O'M and Reddy A K N 1970 In Modern Electrochemistry: An Introduction to an Interdisciplinary Area Vol. 1 (New York: Plenum Press)

36. Dodds W S, Stutzman L F and Sollami B J 1956 Ind. Eng. Chem. 192

37. Stephenson S K, Offeman R D, Robertson G H and Orts W J 2007 Chem. Eng. Sci. 623019 